\title{
Use of a Fluorescent Light Source as an Adjunct to Traditional Methods to Detect Composite Resin Dental Restorations
}

\author{
${ }^{1}$ Dean Kolbinson, ${ }^{2}$ Brendon Reynaud, ${ }^{3}$ Andrew Doig, ${ }^{4}$ Eric Tuttosi, ${ }^{5}$ Alan Heinrichs, ${ }^{6}$ Hyun Lim
}

\begin{abstract}
Aim: To determine if there is a difference in detecting composite restorations by using traditional methods as compared to using an ultraviolet light source and if it would be reasonable to augment the traditional method with the use of an alternate light source.
\end{abstract}

Materials and methods: Twenty-two participants were examined independently by one dentist with traditional visual and tactile dental examination methods (using an overhead light, a dental mirror, and a dental explorer) while simultaneously viewing a pantomograph of the patients and by another dentist utilizing an ultraviolet light source, an overhead light, and an intraoral mirror. The number and position of detected composite restorations for each method was calculated and compared. Comparisons between the two methods were performed using two-sample t-tests. All statistical tests were two-tailed with a significance level of 0.05 .

Results: Differences in the number of surfaces restored with composite restorations $[11.0(\mathrm{SD}= \pm 6.2)$ and $9.6(\mathrm{SD}= \pm 6.4)$ for the manual and light exams respectively] and the number of composite restorations $[8.1(S D= \pm 3.5)$ and $7.1(S D= \pm 3.8)$ for the manual and light exams respectively] between the manual and light examination methods were not statistically significant ( $p$ values $=0.45$ and 0.39 ). Results from the study suggest that the level of agreement between the two examination methods was $81.1 \%$, but that neither method is without the potential for inaccuracy regarding composite restoration detection.

Conclusion and clinical significance: The study indicates that the use of an ultraviolet light source can be an adjunctive clinical tool for the practicing clinical dentist to add to the traditional visual, tactile, and radiographic examination methods, increasing the accuracy and reliability of diagnostic examinations.

Keywords: Agreement, Composite resin, Dental diagnosis, Dental restorations, Detection, Fluorescent light.

How to cite this article: Kolbinson D, Reynaud B, Doig A, Tuttosi E, Heinrichs A, Lim H. Use of a Fluorescent Light Source as an Adjunct to Traditional Methods to Detect Com-

\footnotetext{
${ }^{1,6}$ Professor, ${ }^{2}$ Clinical Instructor, ${ }^{3,4}$ Dentist, ${ }^{5}$ Assistant Professor

${ }^{1-5}$ Department of Pathology, College of Dentistry, University of Saskatchewan, Saskatoon, SK, Canada

${ }^{6}$ Department of Community Health and Epidemiology, College of Medicine, University of Saskatchewan, Saskatoon, SK, Canada

Corresponding Author: Dean Kolbinson, Professor, Department of Pathology, College of Dentistry, University of Saskatchewan, Saskatoon, SK, Canada, e-mail: dean.kolbinson@usask.ca
}

posite Resin Dental Restorations. Int J Experiment Dent Sci 2018;7(2):79-84.

Source of support: Funding for this research was provided by internal funds of the College of Dentistry, University of Saskatchewan to support its student table clinic research program. Conflict of interest: None

\section{INTRODUCTION}

As composite resins continue to advance, amalgam restorations are becoming less abundantly utilized clinically for dental restorative purposes. ${ }^{1}$ Composite resins are used primarily for their esthetic characteristics, and with this, these restorations become increasingly more difficult to detect during diagnostic examinations. ${ }^{2}$ During oral examinations, composite resins can be missed upon inspection due to their effective color matching and can remain completely undetected in the mouth. Also, during removal of composite resins, commonly done due to recurrent decay or defective restoration margins, it is difficult to determine when the restoration is completely removed. $^{3}$

The current ("traditional") method for detecting composite resins is by manual examination (visual and tactile) with the aid of radiographs, commonly pantomographs, also known as panoramic radiographs. ${ }^{4}$ Taking radiographs for the sole purpose of detecting composite restorations is not necessary and may expose the patient to undue radiation, although the amount can be deemed negligible. For example, it would not be unusual for a new patient to have bitewing radiographs and perhaps select periapical radiographs taken, possibly with a pantomograph as well (as opposed to routine or frequent complete mouth series of radiographs). It would not be clinically acceptable to take maxillary anterior periapical radiographs with the sole intent being to determine if a patient has composite restorations that have excellent shade matching. For those types of clinical scenarios, it was wondered if there might be a relatively simple method to detect these (or other) types of composite restorations.

Forensic odontology is the proper handling, examination, and evaluation of dental evidence, which is then presented in the interest of justice. ${ }^{5}$ Forensic odontologists have utilized a method involving detection of compos- 
ite restorations using ultraviolet light sources in order to match restorations found in remains with those in existing dental records. ${ }^{6-10}$ Fluorescent lights, such as the INOVA X5 LED ultraviolet flashlight, produced by Nite Ize, Inc., are affordable ultraviolet light sources and have been shown to be effective in distinguishing composite resins from tooth structure intraorally, as used in forensic odontology. ${ }^{7}$ This light has an optimal wavelength fluorescence of 365-400 nm, which allows composite resins to fluoresce differentially as compared to natural tooth structure. Previous studies have determined that this wavelength has been able to detect composite restorations. ${ }^{711}$ However, composite resins of varying brands and shades fluoresce at different brightnesses and intensities. ${ }^{3}$ Enamel has inherent fluorescence when exposed to ultraviolet light and the detection of resins is based on their fluorescence differing from enamel, regardless of whether the restorations fluoresce more or less, or brighter or dimmer. ${ }^{7}$

Accurately detecting and charting existing composite restorations is an important component of a patient examination, especially with new comprehensive patients. Precisely recording dental findings is also important for dental examinations used for oral health surveys that assist in planning oral health programs and guide oral health overall. ${ }^{12}$ It can be challenging to be confident in determining if all of these restorations have been detected when using the traditional methods. The aim of this preliminary clinical study was to determine if there is a difference in detecting composite restorations by using traditional methods as compared to using an ultraviolet light source and if it would be reasonable to augment the traditional method with the use of an alternate light source.

\section{MATERIALS AND METHODS}

The study received approval from the University of Saskatchewan Research Ethics Board (REB; approval number Bio 15-32). To be eligible for this study, each participant had to meet the following two criteria: all participants must have had current composite restorations, and they all must have had access to an existing panoramic radiograph that was taken since the placement of their last composite restoration. Patients who had recently received an initial examination at the College of Dentistry, University of Saskatchewan and who knowingly had composite restorations and a pantomograph taken at that appointment were contacted. In addition, current students in the college were contacted during the recruitment phase. Only subjects with permanent teeth were recruited. There were no radiographs taken for the sole purpose of this study. All participants signed a consent form which was approved by the REB and completed a medical questionnaire form.

The examinations were conducted by two experienced dentists who were blinded to each other during the examination sessions. Each participant received two examinations at the same session. One was a traditional examination utilizing visual and tactile methods (using a dental mirror and a dental explorer) with the aid of a digital panoramic radiograph. The second examination was completed using an overhead dental light, an intraoral mirror, and the INOVA X5 LED flashlight (produced by Nite Ize, Inc., 5660 Central Avenue, Boulder, CO. 80301, USA); patients were provided with appropriate eye protection during the ultraviolet light examination. One dentist performed all of the traditional examinations while the other performed all of the fluorescent light examinations. A data collection sheet was used for each examination. The findings included the composite restorations identified (by tooth number and surface/s; e.g., tooth \#15 MOD-note that the FDI tooth numbering system was used).

Descriptive statistics were used to summarize the characteristics of the study participants and the composite restorations detected by examination method. Means with standard deviation and medians with ranges of the composite restorations detected by each examination method were then calculated. Comparisons between the two examination methods were performed using twosample t-tests. All statistical tests were two-tailed with a significance level of 0.05 . Sensitivity analysis was also performed to examine the level of agreement between the two examination methods.

\section{RESULTS}

Twenty-three participants enrolled in this study. However, it was determined that the digital pantomograph for one of the subjects was clinically unacceptable and therefore the data for that subject was not included. The remaining 22 participants were used for the study analysis. Of them, 68\% $(n=15)$ were females, and the mean age was $23.5(\mathrm{SD}= \pm 7.2)$ years old. The study participants had varying numbers of teeth with composite restorations (median $=8$, range $1-13$ ). Table 1 shows the baseline demographic characteristics. Table 2 shows the mean number of surfaces restored with composite restorations $[11.0(\mathrm{SD}= \pm 6.2)$ and $9.6(\mathrm{SD}= \pm 6.4)$ for the manual and light exams respectively] and the mean number of composite restorations $[8.1(\mathrm{SD}= \pm 3.5)$ and 7.1 $(\mathrm{SD}= \pm 3.8)$ for the manual and light exams, respectively]. Differences in the number of surfaces restored with composite restorations and the number of composite 
Use of a Fluorescent Light Source as an Adjunct to Traditional Methods to Detect Composite Resin Dental Restorations

\begin{tabular}{ll}
\hline \multicolumn{1}{c}{ Table 1: Summary statistics for 22 participants } \\
\hline Characteristics & $\begin{array}{l}N=22(n, \% ; \text { mean, } \pm S D ; \\
\text { median, range) }\end{array}$ \\
\hline Sex & $7(31.8 \%)$ \\
$\quad$ Male (\%) & $15(68.2 \%)$ \\
Female $(\%)$ & $23.5( \pm 7.2)$ \\
Age & $22.5($ range $14.0-53.0)$ \\
Mean (SD) & \\
Median (range) & \\
Mean number of teeth with & $7.5( \pm 3.3)$ \\
composite restorations & $8.0($ range 1.0-13.0) \\
Mean (SD) & \\
Median (range) & $7.4( \pm 3.5)$ \\
For males & $6.0($ range 2.0-12.0) \\
Mean (SD) & \\
Median (range) & $7.5( \pm 3.3)$ \\
For females & $8.0($ range 1.0 - 13.0) \\
Mean (SD) & \\
Median (range) & \\
\hline SD: Standard devition
\end{tabular}

SD: Standard deviation

Table 3: Frequencies of different types of composite restorations by type of examination method

\begin{tabular}{lll}
\hline & Manual examination & $\begin{array}{l}\text { Light } \\
\text { examination } \\
N=157\end{array}$ \\
\hline Bype of restoration & $N=170$ & $15(9.6 \%)$ \\
O & $13(7.6 \%)$ & $87(55.4 \%)$ \\
D-I-F-L & $94(55.3 \%)$ & \\
D-O & $1(0.6 \%)$ & $12(7.6 \%)$ \\
D-O-B & $10(5.9 \%)$ & \\
F & $1(0.6 \%)$ & \\
I-L & $2(1.2 \%)$ & \\
L & $1(0.6 \%)$ & $2(5.1 \%)$ \\
M-I-F-L & $5(2.9 \%)$ & $1(0.6 \%)$ \\
M-L & $3(1.8 \%)$ & $9(5.7 \%)$ \\
M-O & $3(1.8 \%)$ & $5(3.2 \%)$ \\
M-O-D & $8(4.7 \%)$ & \\
M-O-D-L & $5(2.9 \%)$ & $3(1.9 \%)$ \\
M-O-L & $1(0.6 \%)$ & $7(4.5 \%)$ \\
O-L & $1(0.6 \%)$ & $3(1.9 \%)$ \\
O-B & $16(9.4 \%)$ & $4(2.5 \%)$ \\
D-O-L & $6(3.5 \%)$ & $1(0.6 \%)$ \\
M-I-L & & \\
\hline B DUCCa) & &
\end{tabular}

B, buccal; O, occlusal; D, distal; I, incisal; F, facial; L, lingual; M, mesial restorations between the manual and light examination methods were not statistically significant ( $p$ values $=0.45$ and 0.39 ). A total of 170 composite restorations (with a total of 231 surfaces) was detected by the manual exam and 157 composite restorations (with a total of 210 surfaces) by the light exam. Table 3 itemizes the types of restorations and the frequency with which they were detected by each examination method. The most frequently detected type of composite restoration was occlusal (55.3\% for the manual and $55.4 \%$ for the light exam), followed by buccal restorations $(7.6 \%$ for the manual and $9.6 \%$ for the light exam). Table 4 presents the level of agreement between the two exam methods. Sensitivity is $81.1 \%$, indicating that $81.1 \%$ of the time the light exam came up with the same

Table 2: Number of surfaces restored and restorations by type of exam

\begin{tabular}{llll}
\hline & $\begin{array}{l}\text { Manual } \\
\text { examination } \\
(N=21)\end{array}$ & $\begin{array}{l}\text { Light } \\
\text { examination } \\
(N=22)\end{array}$ & $p$ value \\
Variables & & & \\
\hline Number of restored & & & \\
surfaces & $11.0( \pm 6.2)$ & $9.6( \pm 6.4)$ & 0.45 \\
Mean (SD) & $10.0($ range & $9.0($ range & \\
Median (range) & $1.0-25.0)$ & $1.0-25.0)$ & \\
& & & \\
Number of composite & & & \\
restorations & $8.1( \pm 3.5)$ & $7.1( \pm 3.8)$ & 0.39 \\
Mean (SD) & $8.0($ range 1.0 & $7.0($ range & \\
Median (range) & $-14.0)$ & $1.0-14.0)$ & \\
& & & \\
\hline
\end{tabular}

SD: Standard deviation

$p$ value for 2 -independent samples t-test

conclusion as the manual examination that composite restorations were present. Specificity is $0 \%$, indicating that none of the time the light examination came up with the same conclusion as the manual examination that no composite restoration was present. The kappa agreement value was -0.142 with a $p$ value of 0.06 , indicating that the level of agreement between the manual and light examinations was not statistically significant. Table 5 presents an account of the restorations that were missed by each of the two exam methods. There were 16 composite restorations missed by the manual examination and 28 by the light exam, with the majority of those being occlusal restorations (8 occlusals and one with two separate occlusals missed by the manual examination and 16 occlusals and one with 2 separate occlusals missed by the light examination).

\section{DISCUSSION}

Most of the subjects were young adults, many with several composite restorations (although a small number of participants had only 1 or 2 such restorations). The greatest majority of composite restorations detected involved only the occlusal surface; it is speculated that many of these represented fissure sealants or preventive resin restorations. The level of agreement between the traditional

Table 4: Level of agreement between manual and light examination methods

\begin{tabular}{|c|c|c|c|c|c|}
\hline & & & \multicolumn{3}{|c|}{$\begin{array}{l}\text { Manual } \\
\text { examination }\end{array}$} \\
\hline & & & No & Yes & Total \\
\hline \multirow{4}{*}{$\begin{array}{l}\text { Light } \\
\text { exam- } \\
\text { ination }\end{array}$} & No & Count & 0 & 28 & 28 \\
\hline & & $\begin{array}{l}\% \text { within manual } \\
\text { examination }\end{array}$ & $0.0 \%$ & $18.9 \%$ & $17.1 \%$ \\
\hline & Yes & Count & 16 & 120 & 136 \\
\hline & & $\begin{array}{l}\% \text { within manual } \\
\text { examination }\end{array}$ & $100.0 \%$ & $81.1 \%$ & $82.9 \%$ \\
\hline \multirow[t]{2}{*}{ Total } & & Count & 16 & 148 & 164 \\
\hline & & $\begin{array}{l}\% \text { within manual } \\
\text { examination }\end{array}$ & $100.0 \%$ & $100.0 \%$ & $100.0 \%$ \\
\hline
\end{tabular}




\begin{tabular}{ll}
\hline \multicolumn{2}{c}{ Table 5: Missing cases by examination method } \\
\hline $\begin{array}{l}\text { Missed by manual } \\
\text { examination } \\
N=16\end{array}$ & Missed by light examination \\
O-8 & \\
O, O-1 & O-16 \\
& O, O-1 \\
& OL-1 \\
B-2 & ML-1 \\
& B-2 \\
& IL-1 \\
& MIFL-1 \\
DO-1 & DIFL-1 \\
& DO-2 \\
L-4 & F-2 \\
\hline
\end{tabular}

O, occlusal; L, lingual; M, mesial; B, buccal; I, incisal; F, facial; D, distal method for detecting composite restorations and using a fluorescent light source was moderate, with $81 \%$ sensitivity. This would suggest that neither method is without the potential for inaccurate results. It is speculated that using both of these methods in combination would produce a more accurate outcome.

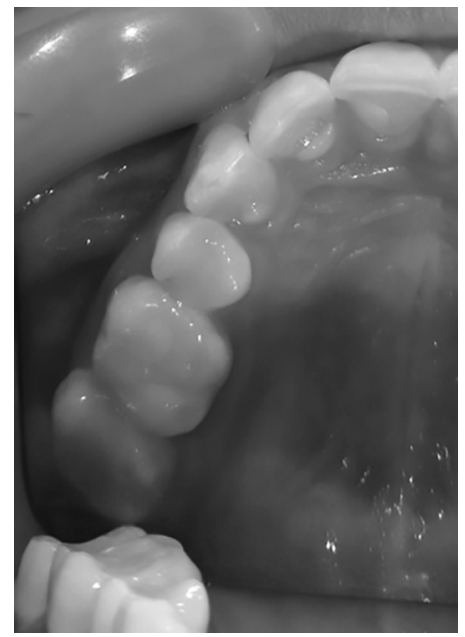

Fig. 1: Occlusal photograph of maxillary left quadrant under overhead dental lighting

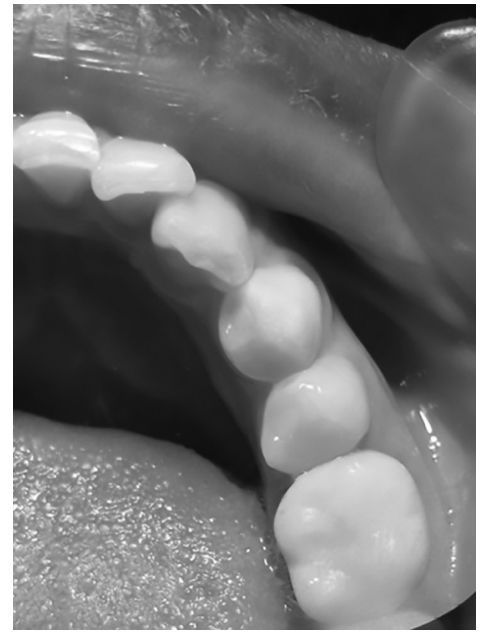

Fig. 3: Occlusal photograph of mandibular left quadrant under overhead dental lighting

Both methods in the study missed several restorations. In some instances, it was evidently clear that there were composite restorations present regardless of which examination method was used. This can be seen in Figures 1 and 2, as the composite restorations on the maxillary first molar are quite evident in both photographs. In other cases, it was easier to detect composite restorations with one examination method as compared to the other. This is exemplified in Figures 3 and 4, in which the occlusal composite restorations could be seen much better under the fluorescent lighting.

The majority of restorations that were missed by both methods were on the occlusal surface, with the next most missed surface using the traditional method being the lingual. The subjects in this study did not happen to have large numbers of composite restorations involving the maxillary or mandibular anterior teeth. There were only 15 such restorations (only 6 involving an interproximal restoration of a maxillary anterior tooth). Of these 15, 6 were missed using the light method, while 3 were

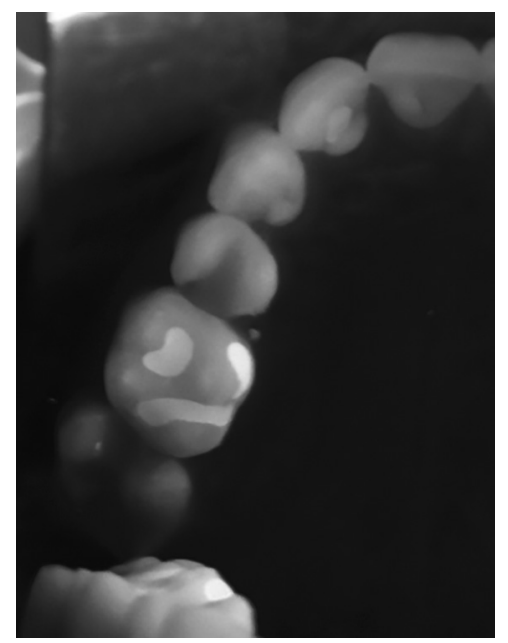

Fig. 2: Occlusal photograph of same maxillary left quadrant under fluorescent lighting

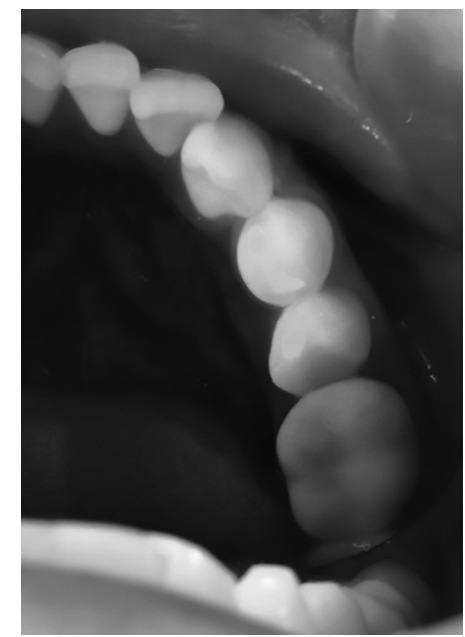

Fig. 4: Occlusal photograph of same mandibular left quadrant under fluorescent lighting 
missed with the traditional method ( 5 were detected by both methods; in one other instance, the light method detected a mesial-incisal-lingual, M-I-L, restoration, while the manual method detected a mesial-lingual, M-L, restoration). Therefore, it is difficult to determine if the use of a fluorescent light would allow more accurate detection of anterior composite restorations.

Previous studies have used a fluorescent light to detect composite restorations (e.g., in forensic settings) $6,7,9,10$ or to help detect composite resin as it is being removed from existing restorations. ${ }^{3}$ A recent study ${ }^{4}$ used images taken with white light or quantitative light-induced fluorescence (QLF-D) technology (Biluminator, Inspektor Research Systems, Amsterdam, The Netherlands) with beginning dental hygiene students. The group using the QLF technology was able to detect restorations (including composites) better than the group assessing only the white-light images. A study by Angelino et al. ${ }^{13}$ used a fluorescent light source to detect dental plaque and observed that the same light accurately identified dental restorations (the types of restorations or other related details were not included).

An empirical observation in this study was that different types of composite restorations had different levels of brightness and were easier to detect than others; this is consistent with a previous study that took place in a laboratory setting. ${ }^{3}$ It would be interesting to better determine the exact cause of the fluorescing properties of the dental materials (e.g., possibly the activating agent camphorquinone) and which of the more recently manufactured composite resin systems are more intensely illuminated with the UV light. It was also observed that one participant, in particular, had a composite restoration that reportedly had not been noted by several previous dentists and was not detected by the traditional method, but was seen using the ultraviolet light method-it was a class VI restoration on a palatal cusp, an uncommon location, but one that was noted with the aid of the UV light. It was also striking on some occasions that composite restorations illuminated so distinctly that it made it seem, at the least, much easier to detect them than using the manual method alone (Fig. 4).

One of the limitations of this study was that there was not a third group that involved a different examiner using both of these methods simultaneously to see if the combination of the two approaches would be superior to either method by itself. However, this was demonstrated in a study by Oh et al. ${ }^{4}$ using a different fluorescent light source in a dental hygiene student training environment.

Another limitation of this study was not using intraoral radiographs. For the purposes of ease of recruitment, it was assumed that it was more likely to find participants with a recent pantomograph as compared to a complete mouth series of radiographs. As interproximal composite restorations in maxillary anterior teeth can be challenging to detect, periapical radiographs of these teeth might have allowed a better rate of restoration detection using the manual method. It is understood that there are a variety of commercially available light systems used for other diagnostic purposes (e.g., caries detection) with similar wavelengths of light. However, no other alternative light sources were used (e.g., LED lights with different wavelengths, curing lights, intraoral camera systems, dental caries diagnostic systems or oral soft tissue lesion detection systems) or filters added to the UV light detection method to see if they also might help detect these restorations or detect them in a superior fashion. There was no attempt to distinguish the different types of composite restorations that were detected in the participants in this study. There was no comparison of attempting to detect composite restorations with the UV light under wet or dry clinical viewing conditions. There was a relatively small sample size and there was no control over the types or number of composite restorations that any individual participant or the collective participant sample had in place. It could be mentioned that there is no absolute certainty that every single composite restoration was detected using either method. The detection of pit and fissure sealants can be a challenge in forensic odontology and more study could be directed at that use of composite resin (some of the composite restorations recorded as part of this study appeared to be sealants). It might be possible in a future study to follow patients from the time they have sealants placed and see if the fluorescent properties of the material change over time.

Both the traditional and UV light methods of detecting composite restorations were relatively accurate, but some restorations were missed using both methods as well. It is suggested that an ultraviolet light source can be a valuable adjunct to the visual, tactile and radiographic method of detecting composite resin restorations.

\section{CONCLUSION AND CLINICAL SIGNIFICANCE}

To detect the presence of composite resin dental restorations in clinical dental patients, the use of an ultraviolet light source is a suitable adjunct to the traditional detection method of visual, tactile and radiographic examination procedures.

\section{ACKNOWLEDGMENT}

The study received approval from the University of Saskatchewan Research Ethics Board (REB; approval number Bio 15-32). 


\section{REFERENCES}

1. Eklund SA. Trends in dental treatment, 1992 to 2007. J Am Dent Assoc 2010;141(4):391-399.

2. Meller C, Klein C. Fluorescence properties of commercial composite resin restorative materials in dentistry. Dent Mater J 2012;31(6):916-923.

3. Bush MA, Hermanson AS, Yetto RJ, et al. The use of ultraviolet LED illumination for composite resin removal: an in vitro study. Gen Dent 2010;58(5):e214-218.

4. Oh HY, Jung HI, Lee JW, et al. Improving the competency of dental hygiene students in detecting dental restorations using quantitative light-induced fluorescence technology. Photodiagnosis and Photodynamic Therapy 2017;17:245-249.

5. Avon SL. Forensic odontology: the roles and responsibilities of the dentist. J Can Dent Assoc 2004;70(7):453-458.

6. Guzy G, Clayton MA. Detection of composite resin restorations using an ultraviolet light-emitting diode flashlight during forensic dental identification. Am J Forensic Med Pathol 2013;34(2):86-89.

7. Hermanson AS, Bush MA, Miller RG, et al. Ultraviolet illumination as an adjunctive aid in dental inspection. J Forensic Sci 2008;53(2):408-411.
8. Carson DO, Orihara Y, Sorbie JL, et al. Detection of white restorative dental materials using an alternative light source. Forensic Sci Int 1997;88(2):163-168.

9. Bush MA, Miller RG, Prutsman-Pfeiffer J, et al. Identification through X-ray fluorescence analysis of dental restorative resin materials: a comprehensive study of noncremated, cremated, and processed-cremated individuals. J Forensic Sci 2007;52(1):157-165.

10. Shakibaie F, Rubinzstein-Dunlop H, Forrest AS, et al. Fluorescence as an aid to recognition of tooth coloured restorations in forensic odontology. Aust Dent J 2012;57(4):S34.

11. Kiran R, Walsh LJ, Forrest A, et al. Forensic applications: fluorescence properties of tooth-coloured restorative materials using a fluorescence DSLR camera. Forensic Sci Int 2017;273:20-28.

12. Tani K, Watari F, Uo M, et al. Discrimination between composite resin and teeth using fluorescence properties. Dental Mater J 2003;22(4):569-580.

13. Angelino K, Shah P, Edlund DA, et al. Clinical validation and assessment of a modular fluorescent imaging system and algorithm for rapid detection and quantification of dental plaque. BMC Oral Health 2017;17(1):162-171. 